
\title{
25 Research Square \\ Efficient Biosynthesis of R-(-)-linalool through Adjusting Expression Strategy and Increasing GPP Supply in Escherichia coli
}

\section{Xun Wang}

Nanjing Forestry University

Jing Wu

Nanjing Forestry University

Jiaming Chen

Nanjing Forestry University

Longjie Xiao

Nanjing Forestry University

Xun Li ( $\nabla$ xunli@njfu.edu.cn )

Nanjing Forestry University https://orcid.org/0000-0002-1267-9263

\section{Research}

Keywords: R-(-)-linalool, Linalool synthase, RBS sequence, Fusion tag, Geranyl diphosphate synthase

Posted Date: December 16th, 2019

DOI: https://doi.org/10.21203/rs.2.18761/v1

License: (c) (1) This work is licensed under a Creative Commons Attribution 4.0 International License. Read Full License

Version of Record: A version of this preprint was published at Journal of Agricultural and Food Chemistry on July 13th, 2020. See the published version at https://doi.org/10.1021/acs.jafc.0c03664. 


\section{Abstract}

Background: R-(-)-linalool is a versatile acyclic monoterpene alcohol with applications in the flavor and fragrance, pharmaceutical, and agrochemical industries. However, plant extraction furnishes only limited and unstable R-(-)-linalool yields that do not satisfy market demand. Therefore, a sustainable yet efficient and productive method of R-(-)-linalool synthesis is urgently needed.

Results: To induce the R-(-)-linalool biosynthesis pathway in E. coli , we expressed heterologous (3R)linalool synthase (LIS) from Lavandula angustifolia (laLIS). We then enhanced R-(-)-linalool production in the cells using a suitable LIS from Streptomyces clavuligerus (bLIS). The bLIS expression was markedly elevated by using optimized ribosomal binding sites (RBSs) and protein fusion tags. R-(-)-linalool output rose from $4.8 \mathrm{mg} \mathrm{L}-1$ to $33.4 \mathrm{mg} \mathrm{L}-1$. To increase the geranyl diphosphate (GPP) content in E. coli, we tested various alterations in geranyl diphosphate synthases (GPPSs) and their mutants. The final E. coli strain harboring GPPS from Abies grandis ( Ag GPPS) accumulated $\leq 100.1 \mathrm{mg} \mathrm{L}-1 \mathrm{R}-(-)$-linalool after 72 $\mathrm{h}$ shake-flask fermentation. This yield gain constitutes a 60.7-fold improvement in R-(-)-linalool biosynthesis over the parent strain. Fed-batch cultivation of the engineered strain in a 1.3-L fermenter yielded 1,027.3 mg L -1 R-(-)-linalool.

Conclusions: In this study, an efficient R-(-)-linalool production pathway was induced in E. colivia the heterologous MVA pathway, AgGPPS, and (3R)-linalool synthase (bLIS). By overexpressing the key enzyme in the engineered $E$. coli strain, R-(-)-linalool production reached $100.1 \mathrm{mg} \mathrm{L}-1$ and 1,027.3 mg L -1 under shake flask- and fed-batch fermentation conditions, respectively. The latter is the highest reported R-(-)-linalool yield to date using an engineered $E$. coli strain. The strategies of key enzyme overexpression and mutation could lay theoretical and empirical foundations for engineering terpenoid pathways and optimizing other metabolic pathways.

\section{Background}

Linalool (3,7-dimethyl-1,6-octadien-3-ol) is an acyclic monoterpene tertiary alcohol and an important natural floral scent. As its hydroxylated third carbon is chiral, in plants it occurs as two enantiomers, namely, (3S)-(+)-linalool (coriandrol) and (3R)-(-)-linalool (licareol). In perfumery, 70\% of the floral scent terpenoids consist of linalool. The odor of this substance is described as "light and refreshing, floralwoody, with a faint citrusy note" [1]. Linalool is a commonly used fragrance and flavoring agent in household detergents, furniture care products, waxes, processed foods, and beverages. Moreover, it is a critical intermediate in vitamin E synthesis. Linalool has antibacterial, anticancer, anti-inflammatory, antioxidant, and antinociceptive properties [2-6]. Worldwide linalool consumption is $\geq 1,000 \mathrm{t} \mathrm{y}^{-1}$ [7]. Linalool occurs in various fruits (e.g. Vitis vinifera L. and Citrus spp.). Recently, it was discovered that certain fungi can produce linalool [8]. R-(-)-linalool has attracted more research attention than synthetic linalool as the former has superior bioactivity. However, the amount of naturally produced R-(-)-linalool is low. In addition, increasing environmental awareness has prompted investigators to generate R-(-)-linalool by more sustainable methods. 
An interesting alternative is the metabolic engineering of microorganisms to produce R-(-)-linalool. This compound is biosynthesized by (3R)-linalool synthase (LIS) from the universal monoterpene precursor geranyl diphosphate (GPP). Two different metabolic pathways have evolved to generate GPP. One is the mevalonate (MVA) pathway in eukaryotes and the archaea. The second is the deoxyxylulose 5-phosphate (DXP) pathway in most prokaryotes and plants (Fig. 1) [9]. Previous studies showed that overexpression of certain native DXP pathway enzymes in E. coli combined with the complete exogenous MVA pathway may efficiently increase the levels of isopentenyl diphosphate (IPP) and its isomer dimethylallyl diphosphate (DMAPP). These molecules are building blocks for GPP [10, 11]. In practice, however, the integration of these strategies limits monoterpene production [12]. To date, the yield of biosynthetic R-(-)linalool has remained very low. We hypothesized that insufficient precursor GPP, low catalytic activity, and inadequate heterologous LIS expression explain these low R-(-)-linalool production rates.

In the present study, we established two-step R-(-)-linalool biosynthesis in recombinant E. coli and improved yield by metabolically engineering LIS and geranyl diphosphate synthase (GPPS). The LIS portion was optimized by screening LISs from various sources and upregulating them. Expression of the LIS portion was modulated by changing the ribosomal binding sites (RBSs) and adding fusion tags to tune R-(-)-linalool production. The GPPS portion was optimized by comparing various GPPSs and their mutants. Fed-batch fermentation with an efficient recombinant $E$. coli strain also improved R-(-)-linalool production. The successful R-(-)-linalool production by E. coli demonstrated here may lay theoretical and empirical foundations for engineering terpenoid pathways and optimizing other metabolic pathways.

\section{Results And Discussion}

\section{Evaluation of the performance of different LISs on R-(-)-linalool production}

In a previous study, the oleaginous yeast Yarrowia lipolytica was constructed. In shake flask cultures, it yielded a maximum linalool titer of $7.0 \pm 0.3 \mathrm{mg} \mathrm{L}^{-1}$ [13]. However, linalool biosynthesis has only had limited success. Its titers have been lower than those for other terpenoids such as geraniol, $\beta$-farnesene, and amorphadiene [14-19]. We propose that the factors contributing to this deficiency include insufficient precursor supply, linalool toxicity, and inefficient LIS. To screen for effective LIS, we compared the performances of those extracted from Lavandula angustifolia (laLIS), Osmanthus fragrans var. thunbergii (ofLIS), and Streptomyces clavuligerus (bLIS) [20]. Truncated laLIS exhibits activity towards its natural substrate GPP $\left(K_{m}=42.7 \pm 4.6\right.$ and $\left.K_{c a t}=3.9 \times 10^{-2}\right)$. Therefore, laLIS may have relatively superior catalytic properties [21]. of LIS is derived from ornamental and medicinal plants and has a strong fragrance. The only known bacterial terpene synthase is bLIS. It produces acyclic compounds and in purified form it uses GPP as a substrate to form R-(-)-linalool $\left(K_{m}=12.9 \pm 1.3\right.$ and $\left.K_{c a t}=8.4 \times 10^{-2}\right)$. Here, the aforementioned genes were codon-optimized and synthesized to avoid any impediments to protein expression. We examined the R-(-)-linalool production profile in $E$. coli strains overexpressing the three optimized LISs. For $72 \mathrm{~h}$, all strains were cultured in 250-mL shake flasks each containing $50 \mathrm{~mL}$ TB 
medium $+5 \mathrm{~g} \mathrm{~L}^{-1}$ glycerol. As shown in Fig. 2a, bLIS expression generated $4.8 \mathrm{mg} \mathrm{L}^{-1} \mathrm{R}$-(-)-linalool, which was a higher concentration than those achieved with laLIS $\left(1.7 \mathrm{mg} \mathrm{L}^{-1}\right)$ or ofLIS (ND). The strain containing bLIS produced certain amounts of nerolidol, as bLIS is both a monoterpene and sesquiterpene synthase. It converts farnesyl diphosphate (FPP) produced by native host-encoded enzymes [22]. For all strains, trace amounts of geraniol were detected in the organic culture overlays but diminished with incubation time. Previous studies showed that $E$. coli bears an unidentified endogenous pathway that converts GPP into geraniol and then dehydrogenates or isomerizes it into other geranoids. Alkaline phosphatase (PhoA) may convert GPP to geraniol [23, 24]. Thus, the biosynthetic pathway for R-(-)linalool production was elucidated via exogenous LIS expression. The catalytic activity of bLIS was higher than those of laLIS and ofLIS. Hence, the bLIS enzyme was selected to enhance R-(-)-linalool production in the subsequent experiments.

R-(-)-linalool formation was accompanied by the abundant generation of indole, which is an intercellular signaling molecule in E. coli (Additional file 1: Fig. S1). We speculate that certain overlooked effects such as an imbalanced metabolic pathway, the accumulation of toxic intermediate metabolites, or certain environmental factors may have stimulated $E$. coli to secrete indole to coordinate their behavior in order to adapt and survive in environmental niches [25-27]. Mevalonate kinase (MK) is a key enzyme that connects the upstream and downstream portions of the MVA pathway. It was regarded as a potential bottleneck [28]. Addition of exogenous MK lowered the indole titer by $50 \%$ (Fig. 2b) and reduced R-(-)linalool production by $17 \%$ (Fig. 2c). Thus, indole formation was associated with the toxic intermediate metabolite HMG-CoA. Moreover, the carbon flux from the upstream to downstream portions of the MVA pathway were not limited by MK overexpression. However, the R-(-)-linalool yield did not increase.

Therefore, certain bottlenecks remained in the R-(-)-linalool synthesis pathway. As bLIS catalyzed the final step in R-(-)-linalool synthesis, sufficient bLIS expression might be required to obtain higher R-(-)-linalool yields.

\section{Modulation of bLIS expression by modification of RBS sequences and application of fusion tags}

Here, bLIS expression was independently regulated at the translational level. This process has been widely used in whole-cell biocatalysis as it is convenient and highly efficient. Bacterial translation processing comprises initiation, elongation, termination, and ribosome turnover. Of these, initiation is the rate-limiting step [29]. Translation initiation can be modulated by modifying RBS sequences [30]. Computational algorithms conveniently design RBS for specific translation initiation rates (TIRs) [29, 31]. In this study, we used the online program "Salis Lab's RBS Calculator" (https://salis.psu.edu/software/) to redesign the bLIS RBS. To control bLIS expression, we designed a set of RBS sequences with TIRs of $100,200,500,5,000,50,000$, and a maximum of 176,000 arbitrary units (au) (Additional file 1: Table S2). The RBS used in pETDuet-bLIS (original plasmid without RBS sequence modification) was predicted to have TIR = 21,711 au. The bLIS had an expected molecular weight (MW) of $37 \mathrm{kDa}$. However, the SDSPAGE analysis disclosed no evidence of target protein overaccumulation in any of the transformants (data not shown). We hypothesized that this outcome was the result of low heterologous protein expression in E. coli. Moreover, there was no indication that bLIS expression increased with TIR strength. 
To elucidate the effect of bLIS expression on R-(-)-linalool production, six different RBS strains were made by transformation with pAGES and pHGFH (Additional file 1: Table S1). The R-(-)-linalool titer increased with TIR strength (Fig. 3a). The RBS with TIR $=50 \mathrm{k}$ au and $176 \mathrm{k}$ au for bLIS contributed to higher R-(-)linalool production levels than the unmodified RBS. WX6600 had the maximum bLIS TIR strength and produced the greatest R-(-)-linalool titer $\left(13.5 \mathrm{mg} \mathrm{L}^{-1}\right)$ after $72 \mathrm{~h}$ cultivation. This rate was 2.8 -fold higher than that determined for the original WX6000. Thus, RBS-based modulation of protein expression level is feasible. Low bLIS TIR strengths (100, 200, and $500 \mathrm{au}$ ) significantly decreased R-(-)-linalool titers and corresponded with poor cell growth (Fig. 3b). The latter, along with low R-(-)-linalool production levels, may have been caused by the abnormal accumulation of toxic IPP and DMAPP when there was insufficient bLIS protein [9].

R-(-)-linalool yield could be improved by modifying the bLIS RBS sequence. However, the R-(-)-linalool titer was lower than expected and there was a theoretical maximum TIR (Additional file 1: Fig. S2). As R-(-)linalool is a secondary metabolite, its actual quantity is very low relative to overall plant biomass. The selection of bLIS from bacteria is conducive to protein solubility and expression in prokaryotic E. coli. Nevertheless, heterologous bLIS expression in E. coli was still very low (Fig. 3c). Therefore, it does not support high R-(-)-linalool production rates and yields. To overcome the bLIS expression barrier, we used fusion tags in the attempt to enhance solubility. Maltose-binding protein (MBP), N-utilization substrate A (NusA), thioredoxin (Trx), and glutathione-Stransferase (GST) are efficient solubility enhancers in E. coli $[32,33]$. Moreover, a peptide $\leq 29$ aa in length derived from the $N$-terminal of chloramphenicol acetyltransferase (CmR29) is an effective solubility enhancer in Synechocystis. Thus, we constructed the fused $b L I S$ genes MBP*bLIS, NuSA*bLIS, GST*bLIS, and CmR29*bLIS. Their measured MW were within expected values: wild type bLIS: 36.3 kDa; MBP*bLIS: 76.6 kDa; NusA*bLIS: 81.2 kDa; GST*bLIS: 61.8 $\mathrm{kDa}$; and CmR29*bLIS: $39.8 \mathrm{kDa}$. Figure $3 \mathrm{c}$ indicates that the use of GST and MBP bLIS fusion tags enhanced bLIS solubility. However, only strain WX6030 expressing the CmR29*bLIS fusion protein presented with augmented R-(-)-linalool yield (16.4 $\left.\mathrm{mg} \mathrm{L}^{-1}\right)$. In contrast, the R-(-)-linalool yields actually diminished in the other three strains (Fig. 3d). A plausible explanation for this result is that although all four fusion tags enhanced soluble bLIS expression, the presence of three large fusion tags ( $>200 \mathrm{aa}$ ) could increase folding interference and alter the biochemical properties of bLIS. On the other hand, the preceding analysis suggested that the short fusion tag CmR29 performed the best. This peptide is shorter than the 50-aa threshold and can drive higher expression levels of heterologous proteins without negatively affecting the three-dimensional structure and catalytic activity of the target enzyme.

The aforementioned findings imply a positive correlation between R-(-)-linalool production and bLIS expression level. The design of RBS sequences and the use of appropriate fusion tags may help surmount the obstacles hindering the generation of high heterologous protein expression levels in E. coli. These tools, then, could be applied towards the regulation of heterologous protein production pathways to improve the yield of target compounds.

\section{Improvement of R-(-)-linalool production by enhancing GPP accessibility}


For strain WX6600 with a modified RBS sequence and strain WX6030 with the fusion protein CmR29*bLIS, the R-(-)-linalool titers were $13.5 \mathrm{mg} \mathrm{L}^{-1}$ and $16.4 \mathrm{mg} \mathrm{L}^{-1}$, respectively. Nevertheless, strain WX6630 with both the modified RBS sequence and the fusion protein CmR29*bLIS produced only 33.4 $\mathrm{mg} \mathrm{L}^{-1} \mathrm{R}-(-)$-linalool. This rate was, in fact, lower than the theoretical $49.4 \mathrm{mg} \mathrm{L}^{-1}$ yield gain. A low GPP level may have accounted for this unsatisfactory output. The DXP and heterogeneous MVA pathways had adequate IPP and DMAPP. However, the GPP pool was very low and a substantial amount of nerolidol by-product was generated. These results were obtained because of the absence of specific, efficient GPPS in the recombinant strains. In E. coli, GPP is produced by the enzyme IspA, which also synthesizes FPP. Therefore, IspA both supplies and competes for the bLIS substrate GPP. To ensure adequate GPP, we selected truncated Abies grandis GPPS (AgGPPS), E. coli IspA and its mutant IspA (S80F), Saccharomyces cerevisiae Erg20, and the Erg20 mutants F96W, N127W, and F96W/N127W and used them to generate R-(-)-linalool [10, 11, 34-36]. Upon co-expression of bLIS, GPPSs, and their mutants, R-(-)-linalool production was altered to varying degrees (Fig. 4a). The co-expression of bLIS and AgGPPS provided the highest R-(-)-linalool yield $\left(100.1 \mathrm{mg} \mathrm{L}^{-1}\right)$. Thus, the major limiting step in the supply of GPP is its conversion from DMAPP/IPP. Erg20 and its mutants have been widely used for terpenoid production by $S$. cerevisiae. However, few studies have reported on Erg20 application for terpenoid synthesis in prokaryotic E. coli. Wild type Erg20 had very low catalytic activity for GPP and FPP generation in E. coli. By changing Phe96 to Trp in Erg20 (F96W), FPP synthase activity was significantly enhanced but the GPP content did not significantly change. Replacing Asn127 with Trp (N127W) increased both GPP and FPP and generated nearly equal amounts of R-(-)-linalool and nerolidol. To obtain relatively higher titers of R-(-)-linalool, the strain WX6636 was induced to express double-mutant Erg20 (F96W/N127W). Its R-(-)linalool and nerolidol yields were $47.0 \mathrm{mg} \mathrm{L}^{-1}$ and $22.4 \mathrm{mg} \mathrm{L}^{-1}$, respectively. The three Erg20 variants showed comparatively higher catalytic activity in E. coli. Nevertheless, the mutant Erg20 (F96W) had the highest FPP synthase activity while the double mutant Erg20 (F96W/N127W) was relatively more conducive to GPP generation (Fig. 4b). IspA (S80F) and AgGPPS had similar performances as precursor suppliers for pinene production in E. coli [37]. In the present study, however, their relative efficacies for R(-)-linalool production were markedly different. Analysis of the fermentation products revealed that 32.3 $\mathrm{mg} \mathrm{L}^{-1}$ and $10.2 \mathrm{mg} \mathrm{L}^{-1}$ nerolidol were obtained from strains WX6632 and WX6637, respectively, after 72 $\mathrm{h}$ shake flask culture (Fig. 4c). Strains WX6632 and WX6637 accumulated total $\mathrm{C}_{5}$ unit concentrations of $0.75 \mathrm{mM}$ and $0.82 \mathrm{mM}$, respectively, after $72 \mathrm{~h}$ shake flask culture. AgGPPS can convert IPP/DMAPP into GPP without generating any FPP by-product. IspA (S80F) can co-synthesize FPP with GPP and bLIS can convert the former into nerolidol. For these reasons, the precursor suppliers IspA (S80F) and AgGPPS achieved different results in pinene and R-(-)-linalool production. Here, we increased R-(-)-linalool production to $100.1 \mathrm{mg} \mathrm{L}^{-1}$ and generated $10.2 \mathrm{mg} \mathrm{L}^{-1}$ nerolidol by using strain WX6637 as its GPP access was relatively greater. Some of the GPP was converted to FPP by the native IspA in E. coli. Further study is required to determine how to eliminate this competing native FPP biosynthesis pathway or enhance bLIS substrate selectivity.

\section{R-(-)-linalool production from an engineered strain in a 1.3-L bioreactor}


Fed-batch fermentation was conducted in a 1.3-L bioreactor to evaluate the overall performance of strain WX6637 which accumulated the highest R-(-)-linalool levels. As illustrated in Fig. 5a, cell growth was rapid starting at $28 \mathrm{~h}$ after the onset of fermentation. The final $\mathrm{OD}_{600}$ at $92 \mathrm{~h}$ was 168.8 . $\mathrm{R}$-(-)-linalool accumulation dramatically increased after $24 \mathrm{~h}$ induction. The final R-(-)-linalool titer was $1,027.3 \mathrm{mg} \mathrm{L}^{-1}$ at $116 \mathrm{~h}$. This R-(-)-linalool yield was the highest ever measured. At that sampling time, the R-(-)-linalool generation rate was $8.86 \mathrm{mg} \mathrm{L}^{-1} \mathrm{~h}^{-1}$. However, the conversion efficiency of glycerol to R-(-)-linalool for strain WX6637 was only $0.012 \mathrm{~g} \mathrm{~g}^{-1}$. Any further R-(-)-linalool production may have been impeded by the toxicity of this substance.

It was proposed that the bacterial toxicity of organic solvents is inversely correlated with their $\log P_{\text {ow }}$. Organic solvents with $\log P_{\text {ow }}=2-5$ are considered toxic to most bacteria. The log $P_{\text {ow }}$ for R-(-)-linalool is 2.97 [38]. A minimum inhibitory concentration assay was run on $\mathrm{R}-(-)$-linalool to quantify its antimicrobial property. E. coli CIBTS1758 was grown in LB medium with $100 \mathrm{mg} \mathrm{L}^{-1}, 200 \mathrm{mg} \mathrm{L}^{-1}, 500 \mathrm{mg} \mathrm{L}^{-1}, 1,000 \mathrm{mg}$ $\mathrm{L}^{-1}$, or 2,000 $\mathrm{mg} \mathrm{L}^{-1}$ exogenous R-(-)-linalool. As shown in Additional file 1: Fig. S3, the culture reached $\mathrm{OD}_{600}=3.1$ after $12 \mathrm{~h}$ incubation in the absence of $\mathrm{R}-(-)$-linalool. $\mathrm{R}-(-)$-linalool concentrations $<1,000 \mathrm{mg}$

$\mathrm{L}^{-1}$ did not significantly inhibit CIBTS1758 growth. However, at R-(-)-linalool concentrations $\geq 1,000 \mathrm{mg} \mathrm{L}$ ${ }^{1}$, CIBTS1758 growth was drastically inhibited. After $12 \mathrm{~h}$, only scant $E$. coli were detected in the culture. The number of colonies on the plates significantly decreased when the R-(-)-linalool titer increased to 1.3 $\mathrm{g} \mathrm{L}^{-1}$ (Fig. 5b). At $\sim 1.4 \mathrm{~g} \mathrm{~L}^{-1}$, R-(-)-linalool had reduced the number of surviving bacterial colonies by $50 \%$ relative to the untreated (Fig. 5c). Two-phase cultures may partially alleviate R-(-)-linalool inhibition. However, substantial improvements in the tolerance of $E$. coli to organic solvents and microbial terpenoid production will require considerable metabolic and protein engineering and fermentation optimization.

\section{Conclusions}

Here, R-(-)-linalool production via recombinant E. coli strains was optimized by systematic and rational approaches (Fig. 6). The effects of various LISs on R-(-)-linalool generation were compared. A maximum $\mathrm{R}-(-)$-linalool titer of $4.8 \mathrm{mg} \mathrm{L}^{-1}$ was obtained by expressing bLIS in recombinant E. coli. Approximate 2.8fold and 3.4-fold gains in R-(-)-linalool production (13.5 $\mathrm{mg} \mathrm{L}^{-1}$ and $16.4 \mathrm{mg} \mathrm{L}^{-1}$ ) were realized when the rate-limiting enzyme bLIS was overexpressed by using a modified RBS sequence and the CmR29 fusion tag, respectively. The combination of the strongest RBS and the CmR29 fusion tag caused $E$. coli WX6630 to produce $37.5 \mathrm{mg} \mathrm{L}^{-1} \mathrm{R}$-(-)-linalool. To increase GPP accessibility for R-(-)-linalool synthesis, several GPPSs and their mutants were tested. WX6637 containing AgGPPS accumulated R-(-)-linalool $\leq$ $100.1 \mathrm{mg} \mathrm{L}^{-1}$ with minimal by-products after $72 \mathrm{~h}$ shake-flask culture. After $116 \mathrm{~h}$ fed-batch fermentation, WX6637 generated 1,027.3 $\mathrm{mg} \mathrm{L}^{-1} \mathrm{R}-(-)-$-linalool. To the best of our knowledge, the R-(-)-linalool yield in the present study is the highest titer reported thus far. R-(-)-linalool was significantly toxic to $E$. colicells at titers $\geq 1.3 \mathrm{~g} \mathrm{~L}^{-1}$. R-(-)-linalool toxicity could be partially attenuated by two-phase culture. Nevertheless, R(-)-linalool toxicity to $E$. coli remains an impediment to higher R-(-)-linalool yield. Future research should focus on augmenting R-(-)-linalool tolerance in E. coli. Furthermore, enhancements to real-time product 
separation may help increase the economic feasibility and practicality of microbial R-(-)-linalool production. The outcome of the present study lays theoretical and empirical foundations for the production of other useful compounds via recombinant $E$. coli strains.

\section{Methods}

\section{Chemicals and materials}

Restriction enzymes, T4 DNA ligase, T4 polynucleotide kinase, PrimeSTAR Max DNA polymerase, and PCR reagents were purchased from Takara (Dalian, China). The primers were synthesized by Sangon (Shanghai, China). The corresponding codon-optimized genes were chemically synthesized by Generay (Shanghai, China). The gel extraction, PCR purification, and plasmid mini kits were purchased from Axygen Scientific (Suzhou, China). All other chemicals used were of the highest available purity.

\section{Plasmid construction}

The primers used for the genetic modifications are listed in Additional file 1: Table S4. The plasmids pHGFH and pAGES containing expression cassettes for the partial DXP and full-length MVA pathway genes were gifts from Prof. Sheng Yang. The codon-optimized LIS genes from Lavandula angustifolia (Genbank No. ABB73045.1), Osmanthus fragrans var. thunbergii (Genbank No. ABB73045.1), and Streptomyces clavuligerus (Genbank No. D5SL78.1) were chemically synthesized with codons optimized for Escherichia coli expression. The LIS gene was cloned between the $\mathrm{Ncol}$ and $\mathrm{BamHI}$ sites of the pETDuet-tac vector. The plasmid products were pETDuet-IaLIS, pETDuet-of $L I S$, and pETDuet- $b L I S$. The gene encoding MK was cloned downstream of $b L I S$ on pETDuet- $b L I S$ and yielded the pETDuet- $b L I S-M K$. The RBS sequences with various TIRs were calculated by Sails Lab [29]. The RBS sequences with TIRs of $100,200,500,5,000,50,000$, and 176,000 au were added to the $5^{\prime}$-end of $b L I S$ in pETDuet- $b L I S$. The plasmid products were pETDuet- $b L I S(0.1 \mathrm{k})$, pETDuet- $b L I S(0.2 \mathrm{k})$, pETDuet- $b L I S(0.5 \mathrm{k}), \mathrm{pETDuet}-b L I S(5$ k), pETDuet-bLIS (50 k), and pETDuet-bLIS (max). The MBP, NuSA, CmR29, and GST fusion tag genes were chemically synthesized and subcloned into the upstream region of $b L I S$ in pETDuet- $b L I S$ with a ClonExpress II one-step cloning kit (Vazyme Biotech Co. Ltd., Nanjing, China). The plasmid products were pETDuet-MBP*bLIS, pETDuet-NuSA*bLIS, pETDuet-CmR29*bLIS, and pETDuet-GST*bLIS. The optimized RBS sequence was ligated into the 5 '-end of fused $C m R 29 * b L I S$ and generated pETDuet-CmR29*bLIS (maximum). The gene encoding GPP synthase from Abies grandis (Genbank No. AAN01134.1) was truncated by 86 aa from its $N$-terminus, codon-optimized for $E$. coli, and inserted behind the second tac promoter of pETDuet-CmR29*bLIS (max). The plasmid product was pETDuet-CmR29*bLIS (max)AgGPPS. The plasmids pETDuet-CmR29*bLIS (max)-IspA and pETDuet-CmR29*bLIS (max)-Erg20 were constructed based on pETDuet-Cm29R*bLIS (max)-AgGPPS by replacing AgGPPS with IspA from E. coli and Erg20 from Saccharomyces cerevisiae. The mutants of IspA (S80F), Erg20 (F96W), Erg20 (N127W), and Erg20 (F96W/N127W) were obtained by inverse PCR and construction of pETDuet-CmR29*bLIS (max)-IspA (S80F), pETDuet-CmR29*bLIS (max)-Erg20 (F96W), pETDuet-CmR29*bLIS (max)-Erg20 
(N127W), and pETDuet-CmR29*bLIS (max)-Erg20 (F96W/N127W). The DNA sequences of all constructs were verified by full re-sequencing.

\section{Strains and culture conditions}

All plasmids and strains constructed here are listed in Additional file 1: Table S1. For R-(-)-linalool production, the seed culture was incubated at $37^{\circ} \mathrm{C}$ overnight in Luria-Bertani (LB) medium $\left(10 \mathrm{~g} \mathrm{~L}^{-1}\right.$ tryptone, $5 \mathrm{~g} \mathrm{~L}^{-1}$ yeast extract, and $10 \mathrm{~g} \mathrm{~L}^{-1}$ sodium chloride) with antibiotics (100 mg L-1 ampicillin, $50 \mathrm{mg}$ $\mathrm{L}^{-1}$ spectinomycin, and $34 \mathrm{mg} \mathrm{L}^{-1}$ chloramphenicol). Shake flask cultures were initiated by inoculating 0.5 $\mathrm{mL}$ seed culture into $50 \mathrm{~mL}$ TB medium $\left(12 \mathrm{~g} \mathrm{~L}^{-1}\right.$ tryptone, $24 \mathrm{~g} \mathrm{~L}^{-1}$ yeast extract, $9.4 \mathrm{~g} \mathrm{~L}^{-1} \mathrm{~K}_{2} \mathrm{HPO}_{4}$, and

$\left.2.2 \mathrm{~g} \mathrm{~L}^{-1} \mathrm{KH}_{2} \mathrm{PO}_{4}\right)$ containing $0.5 \%(\mathrm{v} / \mathrm{v})$ glycerol as the main carbon source. When $\mathrm{OD}_{600} \sim 0.8-1,0.5 \mathrm{mM}$ isopropyl $\beta$-D-1-thiogalactopyranoside (IPTG) was added and $10 \%(\mathrm{v} / \mathrm{v})$ isopropyl myristate was overlaid on the medium to prevent the loss of volatile products. After induction, the temperature was decreased to $25{ }^{\circ} \mathrm{C}$. The experiments were run in duplicate. The $\mathrm{R}-(-)-$-linalool concentration and $\mathrm{OD}_{600}$ were quantified every $24 \mathrm{~h}$.

\section{Product analysis by GC-MS and GC-FID}

One milliliter of uniformly mixed culture broth with the isopropyl myristate phase was sampled and concentrated by centrifuging at $13,500 \times \mathrm{g}$ for $5 \mathrm{~min}$. The isopropyl myristate layer was analyzed by GCFID and GC-MS. R-(-)-linalool, geraniol, and nerolidol were identified by a Trace GC 2000 Ultra (Thermo Fisher Scientific, Waltham, MA, USA) fitted with a DB-5 column $(30 \mathrm{~m} \times 0.25 \mathrm{~mm} \times 0.25 \mu \mathrm{m})$ and a Thermo Finnigan Trace DSQ mass spectrometer operating with electron ionization (ion source temperature: $200^{\circ} \mathrm{C}$; electron energy: $70 \mathrm{eV}$ ). The scan rate was set to $500 \mathrm{amu} \mathrm{s}{ }^{-1}$. The operating parameters were as follows: injector: $220^{\circ} \mathrm{C}$; detector: $250^{\circ} \mathrm{C}$; oven temperature: start at $40^{\circ} \mathrm{C}$, hold for 1 min, programmed to rise from $40{ }^{\circ} \mathrm{C}$ to $240{ }^{\circ} \mathrm{C}$ at $10{ }^{\circ} \mathrm{C} \mathrm{min}^{-1}$, hold for $5 \mathrm{~min}$; helium carrier gas flow: 1.0 $\mathrm{mL} \mathrm{min}{ }^{-1}$; injection mode: $1 \mathrm{~mL}$ sample with splitless injection. Monoterpene and sesquiterpene identities were confirmed by comparing mass spectra and retention times against authentic standards.

Chiral gas chromatography was performed to determine enantiomeric linalool distribution. The isopropyl myristate phase was analyzed on a Cyclosil-B $\beta$-cyclodextrin chiral capillary column $(30 \mathrm{~m} \times 0.25 \mathrm{~mm} \times$ $0.25 \mu \mathrm{m}$ ). Samples were injected at $200{ }^{\circ} \mathrm{C}$ into a GC (No. 7890A; Agilent Technologies, Santa Clara, CA, USA) fitted with a flame ionization detector (FID). The oven temperature was first held at $80^{\circ} \mathrm{C}$ for 5 min and gradually increased at a rate of $5{ }^{\circ} \mathrm{C} \mathrm{min}-1$ to $200{ }^{\circ} \mathrm{C}$ where it was held for another $5 \mathrm{~min}$. The detector temperature was maintained at $260^{\circ} \mathrm{C}$. Product identity was confirmed by comparison against authentic R-(-)-linalool standards (Additional file 1: Fig. S4).

\section{Protein analysis}

To establish the expression levels and solubilities of the fused bLISs, pETDuet- $b L I S, \mathrm{pETDuet}-M B P * b L I S$, pETDuet-NusA*bLIS, pETDuet-Cm29*bLIS, and pETDuet-GST*bLIS were transformed into $E$. coli BL21 to 
yield the recombinant strains WX6009, WX6019, WX6029, WX6039, and WX6049, respectively. Heterologous expression of the fused bLISs in E. coli BL21 was compared by $12 \%$ SDS-PAGE analysis of the crude protein extracts.

\section{Fed-batch fermentation in a 1.3-L bioreactor}

The process used for R-(-)-linalool production was adapted from a previously published method [18]. A single colony of strain WX6637 was incubated overnight in $5 \mathrm{~mL} \mathrm{LB}$ medium at $37^{\circ} \mathrm{C}$ and rotated at 200 $\mathrm{rpm}$. Then $40 \mathrm{~mL}$ LB medium ( $10 \% \mathrm{v} / \mathrm{v})$ was inoculated with this pre-culture. After $6-8 \mathrm{~h}$ cultivation (37 ${ }^{\circ} \mathrm{C} ; 200 \mathrm{rpm}$ ) and at $\mathrm{OD}_{600}=5$, the pre-culture was then used to inoculate the main culture. Fed-batch fermentation experiments were conducted in a 1.3-L bioreactor (New Brunswick Scientific BioFlo/CelliGen 115, Edison, NJ, USA). At the start of the batch phase, the bioreactor contained $0.4 \mathrm{~L} \mathrm{AM}$ mineral medium (4.2 $\mathrm{g} \mathrm{L}^{-1} \mathrm{KH}_{2} \mathrm{PO}_{4}, 15.7 \mathrm{~g} \mathrm{~L}^{-1} \mathrm{~K}_{2} \mathrm{HPO}_{4} \cdot 3 \mathrm{H}_{2} \mathrm{O}, 2 \mathrm{~g} \mathrm{~L}^{-1}\left(\mathrm{NH}_{4}\right)_{2} \mathrm{SO}_{4}, 1.7 \mathrm{~g} \mathrm{~L}^{-1}$ citric acid, $8.4 \mathrm{mg} \mathrm{L}^{-1}$ EDTA, $15 \mathrm{~g} \mathrm{~L}^{-}$ 1 glycerol, $1.2 \mathrm{~g} \mathrm{~L}^{-1} \mathrm{MgSO}_{4} \cdot 7 \mathrm{H}_{2} \mathrm{O}, 4.5 \mathrm{mg} \mathrm{L}^{-1}$ thiamine $\mathrm{HCl}$, and $10 \mathrm{~mL} \mathrm{~L}^{-1}$ trace metal solution), $100 \mathrm{mg} \mathrm{L}^{-1}$ ampicillin, $50 \mathrm{mg} \mathrm{L}^{-1}$ spectinomycin, and $34 \mathrm{mg} \mathrm{L}^{-1}$ chloramphenicol. The trace metal solution consisted of $0.25 \mathrm{~g} \mathrm{~L}^{-1} \mathrm{CoCl}_{2} \cdot 6 \mathrm{H}_{2} \mathrm{O}, 1.5 \mathrm{~g} \mathrm{~L}^{-1} \mathrm{MnCl}_{2} \cdot 4 \mathrm{H}_{2} \mathrm{O}, 0.15 \mathrm{~g} \mathrm{~L}^{-1} \mathrm{CuCl}_{2} \cdot 2 \mathrm{H}_{2} \mathrm{O}, 0.3 \mathrm{~g} \mathrm{~L}^{-1} \mathrm{H}_{3} \mathrm{BO}_{4}, 0.25 \mathrm{~g} \mathrm{~L}^{-1}$ $\mathrm{Na}_{2} \mathrm{MoO}_{4} \cdot 2 \mathrm{H}_{2} \mathrm{O}, 1.3 \mathrm{~g} \mathrm{~L}^{-1} \mathrm{Zn}\left(\mathrm{CH}_{3} \mathrm{COO}\right)_{2} \cdot 2 \mathrm{H}_{2} \mathrm{O}$, and $10 \mathrm{~g} \mathrm{~L}^{-1} \mathrm{Fe}(\mathrm{III})$ citrate hydrate. Fermentation was run at $37^{\circ} \mathrm{C}$ and $25 \%(\mathrm{v} / \mathrm{v})$ ammonia or $10 \%(\mathrm{v} / \mathrm{v}) \mathrm{HCl}$ was used to maintain the $\mathrm{pH}$ at 7.0. The former also served as a nitrogen source during fermentation. The dissolved oxygen (DO) concentration was maintained at $>15 \%$ saturation by increasing the stirring speed from $200 \mathrm{rpm}$ to a maximum of $700 \mathrm{rpm}$ and by aeration with 3 vvm air. Antifoam 204 (Sigma-Aldrich Corp., St. Louis, MO, USA) was added to prevent foaming. When the initial glycerol in the medium was nearly depleted $\left(<0.1 \mathrm{~g} \mathrm{~L}^{-1}\right)$ and the DO spiked, a glycerol concentration-based feed was activated by adding a solution at an appropriate rate to maintain the glycerol concentration $<5 \mathrm{~g} \mathrm{~L}^{-1}$. The feed solution consisted of $650 \mathrm{~g} \mathrm{~L}^{-1}$ glycerol, $12 \mathrm{~g} \mathrm{~L}^{-1}$ $\mathrm{MgSO}_{4} \cdot 7 \mathrm{H}_{2} \mathrm{O}, 10.7 \mathrm{~g} \mathrm{~L}^{-1}\left(\mathrm{NH}_{4}\right)_{2} \mathrm{SO}_{4}, 13 \mathrm{mg} \mathrm{L}^{-1} \mathrm{EDTA}$, and $10 \mathrm{~mL} \mathrm{~L}^{-1}$ feed trace metal solution $\left(0.4 \mathrm{~g} \mathrm{~L}^{-1}\right.$ $\mathrm{CoCl}_{2} \cdot 6 \mathrm{H}_{2} \mathrm{O}, 2.35 \mathrm{~g} \mathrm{~L}^{-1} \mathrm{MnCl}_{2} \cdot 4 \mathrm{H}_{2} \mathrm{O}, 0.25 \mathrm{~g} \mathrm{~L}^{-1} \mathrm{CuCl}_{2} \cdot 2 \mathrm{H}_{2} \mathrm{O}, 0.5 \mathrm{~g} \mathrm{~L}^{-1} \mathrm{H}_{3} \mathrm{BO}_{4}, 0.4 \mathrm{~g} \mathrm{~L}^{-1} \mathrm{Na}_{2} \mathrm{MoO}_{4} \cdot 2 \mathrm{H}_{2} \mathrm{O}, 1.6 \mathrm{~g}$ $\mathrm{L}^{-1} \mathrm{Zn}\left(\mathrm{CH}_{3} \mathrm{COO}\right)_{2} \cdot 2 \mathrm{H}_{2} \mathrm{O}$, and $4 \mathrm{~g} \mathrm{~L}^{-1} \mathrm{Fe}(\mathrm{III})$ citrate hydrate). R-(-)-linalool production was induced with 0.5 $\mathrm{mM} I P T G$ at $\mathrm{OD}_{600}=35$. Then $10 \%(\mathrm{v} / \mathrm{v})$ isopropyl myristate was added to the medium to extract the product in situ with a biocompatible organic phase. The post-induction culture temperature was held at $30{ }^{\circ} \mathrm{C}$. Samples were drawn throughout fermentation to measure cell density and the glycerol and R-(-)linalool concentrations. Glycerol was analyzed with a glycerol assay kit (Jiancheng, Nanjing, China) according to the manufacturer's instructions.

\section{R-(-)-linalool toxicity assay}

To evaluate the toxicity of R-(-)-linalool to E. coli CIBTS1758, a 12-h seed culture was diluted with $1 \mathrm{~mL}$ fresh $L B$ medium $\left(\mathrm{OD}_{600}=0.2\right)$ containing $100,200,500,1,000$, or $2,000 \mathrm{mg} \mathrm{L}^{-1} \mathrm{R}$-(-)-linalool. Two hundred-microliter samples were transferred to 96 -well plates and incubated with continuous shaking at $37^{\circ} \mathrm{C}$ in a Biotek Cytation 3 imaging plate reader (Biotek Instruments Inc., Winooski, VT, USA). OD 600 was 
automatically measured every $0.5 \mathrm{~h}$. To determine the minimum inhibitory concentrations of R-(-)-linalool, equal amounts of cells at $\mathrm{OD}_{600}=1$ were subjected to serial 10 -fold dilutions with fresh LB medium.

Then $100 \mu \mathrm{L}$ of a $10^{-5}$ dilution was spread onto inhibitory LB plates containing $0,1,1.1,1.2,1.3,1.4$, or 1.5 $\mathrm{g} \mathrm{L}^{-1} \mathrm{R}$-(-)-linalool and incubated for $12 \mathrm{~h}$ at $37^{\circ} \mathrm{C}$. The viable cells on each plate were then counted. Three independent biological / experimental replicates were prepared. The minimum inhibitory concentration of $\mathrm{R}-(-)-$ linalool was defined as that which reduced colony formation by $\leq 2 \times$ on inhibitory LB plates.

\section{Abbreviations}

AA-CoA, acetoacetyl-CoA; A-CoA, acetyl-CoA; au, arbitrary units; CDP-ME, 4-diphosphocytidyl-2-Cmethylerythritol; CDP-MEP, 4-diphosphocytidyl-2-C-methyl-D-erythritol 2-phosphate; DMAPP, dimethylallyl diphosphate; DXP, deoxyxylulose 5-phosphate; FPP, farnesyl diphosphate; G-3-P: glyceraldehyde 3phosphate; GC-FID, gas chromatography-flame ionization detection; GC-MS, gas chromatography-mass spectrometry; GPP, geranyl diphosphate; GPPS, geranyl diphosphate synthase; GST, glutathione-Stransferase; HMBPP: (E)-4-hydroxy-3-methyl-but-2-enyl-diphosphate; HMG-CoA: 3-hydroxy-3methylglutaryl-CoA; IPP, isopentenyl diphosphate; IPTG, isopropyl $\beta$-d-1-thiogalactopyranoside; LB, LuriaBertani medium; LIS, linalool synthase; MBP, maltose-binding protein; MEcPP: 2-C-methyl-D-erythritol 2,4cyclodiphosphate; MEP: 2-C-methylerythritol 4-phosphate; Mev-P, mevalonate-5-phosphate; Mev-PP, mevalonate-5-diphosphate; MK, mevalonate kinase; MVA, mevalonate; NusA, N-utilization substrate A; PCR, polymerase chain reaction; RBS, ribosomal binding site; SDS-PAGE, sodium dodecyl sulfate polyacrylamide gel electrophoresis; TIR, translation initiation rate; Trx, thioredoxin (Trx)

\section{Declarations}

\section{Ethics approval and consent to participate}

Not applicable.

\section{Consent for publication}

Not applicable.

\section{Availability of data and materials}

The datasets used and/or analyzed during the current study are available from the corresponding author on reasonable request.

\section{Competing interests}

The authors declare that they have no competing interests.

\section{Funding}

This work was financially supported by the National Key Research \& Development Program of China (2017YFD0600205), the National Natural Science of China (No. 21878160), and the Topnotch Academic Programs Project (TAPP) Education Institutions.

\section{Authors' contributions}

$X L$ and XW conceived and designed the experiments. XW, JW JMC and LJX did the lab work, plasmid construction, strain screening and cultivation, fermentation, and product detection. XW did the literature 
review and prepared the manuscript. XL, JW JMC and LJX helped to revise the manuscript. All authors read and approved the final manuscript.

\section{Acknowledgements}

We thank Prof. Sheng Yang at Chinese Academy of Sciences for the MVA gene (CIBTS1758 and pAGES, pHGFH). We would like to thank Editage (www.editage.com) for English language editing.

Authors' information

1 Jiangsu Provincial Key Lab for the Chemistry and Utilization of Agro-forest Biomass, Nanjing Forestry University, Nanjing 210037, China

2 Jiangsu Key Laboratory of Biomass-based Green Fuels and Chemicals, Nanjing Forestry University, Nanjing 210037, China

3 College of Chemical Engineering, Nanjing Forestry University, Nanjing 210037, China

\section{Additional Files}

Additional file 1: Fig. S1 Formation of R-(-)-linalool was accompanied by production of approximately 50 $\mathrm{mg} / \mathrm{L}$ indole. Fig. S2 Positive correlation between R-(-)-linalool production and bLIS expression level, especially when the TIR strength of bLIS is between $5 \mathrm{k}$ au and theoretical maximum 176k au. Fig. S3 $\mathrm{OD}_{600}$ value of $E$. coli CIBTS1758 which were cultivated in LB medium containing different concentration of R-(-)-linalool for $12 \mathrm{~h}$, samples were automatically measured each $0.5 \mathrm{~h}$. Fig. S4 Chiral phase GC and GC-MS identification of R-(-)-linalool product of LIS. Table S1 Plasmids and strains used in this study. Table S2 Different TIRs strength and corresponding RBS sequences. Table S3 Sequences of fusion tags used in this study. Table S4 Primers used in this study. Table S5 Genebank ID and sequences of LISs and GPPSs used in this study.

\section{References}

1. Stashenko EE, Martinez JR: Sampling flower scent for chromatographic analysis. Journal of Separation Science 2008, 31:2022-2031.

2. Peana AT, Marzocco S, Popolo A, Pinto A: (-)-Linalool inhibits in vitro NO formation: Probable involvement in the antinociceptive activity of this monoterpene compound. Life Sciences 2006, 78:719-723.

3. Peana AT, Rubattu P, Piga GG, Fumagalli S, Boatto G, Pippia P, De Montis MG: Involvement of adenosine A1 and A2A receptors in (-)-linalool-induced antinociception. Life Sciences 2006, 78:24712474.

4. Herman A, Tambor K, Herman A: Linalool Affects the Antimicrobial Efficacy of Essential Oils. Current Microbiology 2016, 72:165-172.

5. Aprotosoaie AC, Hancianu M, Costache I-I, Miron A: Linalool: a review on a key odorant molecule with valuable biological properties. Flavour and Fragrance Journal 2014, 29:193-219. 
6. Beier RC, Byrd JA, II, Kubena LF, Hume ME, McReynolds JL, Anderson RC, Nisbet DJ: Evaluation of linalool, a natural antimicrobial and insecticidal essential oil from basil: Effects on poultry. Poultry Science 2014, 93:267-272.

7. Lapczynski A, Letizia CS, Api AM: Addendum to Fragrance material review on linalool. Food and Chemical Toxicology 2008, 46:S190-S192.

8. Linalool S: Initial Assessment Report For SIAM 14. OECD SIDS, Paris, France; 2002.

9. Martin VJJ, Pitera DJ, Withers ST, Newman JD, Keasling JD: Engineering a mevalonate pathway in Escherichia coli for production of terpenoids. Nature Biotechnology 2003, 21:796-802.

10. Zhou J, Wang C, Yoon S-H, Jang H-J, Choi E-S, Kim S-W: Engineering Escherichia coli for selective geraniol production with minimized endogenous dehydrogenation. Journal of Biotechnology 2014, 169:42-50.

11. Alonso-Gutierrez J, Chan R, Batth TS, Adams PD, Keasling JD, Petzold CJ, Lee TS: Metabolic engineering of Escherichia coli for limonene and perillyl alcohol production. Metabolic Engineering 2013, 19:33-41.

12. Sarria S, Wong B, Martin HG, Keasling JD, Peralta-Yahya P: Microbial Synthesis of Pinene. Acs Synthetic Biology 2014, 3:466-475.

13. Cao X, Wei L-J, Lin J-Y, Hua Q: Enhancing linalool production by engineering oleaginous yeast Yarrowia lipolytica. Bioresource Technology 2017, 245:1641-1644.

14. Deng Y, Sun M, Xu S, Zhou J: Enhanced (S)-linalool production by fusion expression of farnesyl diphosphate synthase and linalool synthase in Saccharomyces cerevisiae. Journal of Applied Microbiology 2016, 121:187-195.

15. Liu W, Xu X, Zhang R, Cheng T, Cao Y, Li X, Guo J, Liu H, Xian M: Engineering Escherichia coli for highyield geraniol production with biotransformation of geranyl acetate to geraniol under fed-batch culture. Biotechnology for Biofuels 2016, 9:58.

16. Jiang G-Z, Yao M-D, Wang Y, Zhou L, Song T-Q, Liu H, Xiao W-H, Yuan Y-J: Manipulation of GES and ERG20 for geraniol overproduction in Saccharomyces cerevisiae. Metabolic Engineering 2017, 41:5766.

17. Meadows AL, Hawkins KM, Tsegaye Y, Antipov E, Kim Y, Raetz L, Dahl RH, Tai A, MahatdejkulMeadows T, Xu L, et al: Rewriting yeast central carbon metabolism for industrial isoprenoid production. Nature 2016, 537:694-697.

18. Tsuruta H, Paddon CJ, Eng D, Lenihan JR, Horning T, Anthony LC, Regentin R, Keasling JD, Renninger NS, Newman JD: High-Level Production of Amorpha-4,11-Diene, a Precursor of the Antimalarial Agent Artemisinin, in Escherichia coli. Plos One 2009, 4(2):e4489.

19. Westfall PJ, Pitera DJ, Lenihan JR, Eng D, Woolard FX, Regentin R, Horning T, Tsuruta H, Melis DJ, Owens A, et al: Production of amorphadiene in yeast, and its conversion to dihydroartemisinic acid, precursor to the antimalarial agent artemisinin. Proceedings of the National Academy of Sciences of the United States of America 2012, 109:111-118. 
20. Nakano C, Kim H-K, Ohnishi Y: Identification and characterization of the linalool/nerolidol synthase from Streptomyces clavuligerus. Chembiochem : a European journal of chemical biology 2011, 12:2403-2407.

21. Landmann C, Fink B, Festner M, Dregus M, Engel K-H, Schwab W: Cloning and functional characterization of three terpene synthases from lavender (Lavandula angustifolia). Archives of Biochemistry and Biophysics 2007, 465:417-429.

22. Karuppiah V, Ranaghan KE, Leferink NGH, Johannissen LO, Shanmugam M, Cheallaigh AN, Bennett NJ, Kearsey LJ, Takano E, Gardiner JM, et al: Structural Basis of Catalysis in the Bacterial Monoterpene Synthases Linalool Synthase and 1,8-Cineole Synthase. Acs Catalysis 2017, 7:62686282.

23. Leferink NGH, Jervis AJ, Zebec Z, Toogood HS, Hay S, Takano E, Scrutton NS: A 'Plug and Play' Platform for the Production of Diverse Monoterpene Hydrocarbon Scaffolds in Escherichia coli. Chemistryselect 2016, 1:1893-1896.

24. Liu W, Zhang R, Tian N, Xu X, Cao Y, Xian M, Liu H: Utilization of alkaline phosphatase PhoA in the bioproduction of geraniol by metabolically engineered Escherichia coli. Bioengineered 2015, 6:288293.

25. Ajikumar PK, Xiao W-H, Tyo KEJ, Wang Y, Simeon F, Leonard E, Mucha O, Phon TH, Pfeifer B, Stephanopoulos G: Isoprenoid Pathway Optimization for Taxol Precursor Overproduction in Escherichia coli. Science 2010, 330:70-74.

26. Pitera DJ, Paddon CJ, Newman JD, Keasling JD: Balancing a heterologous mevalonate pathway for improved isoprenoid production in Escherichia coli. Metabolic Engineering 2007, 9:193-207.

27. Thi Hiep H, Lee J-H, Cho MH, Wood TK, Lee J: Environmental factors affecting indole production in Escherichia coli. Research in Microbiology 2011, 162:108-116.

28. Anthony JR, Anthony LC, Nowroozi F, Kwon G, Newman JD, Keasling JD: Optimization of the mevalonate-based isoprenoid biosynthetic pathway in Escherichia coli for production of the antimalarial drug precursor amorpha-4,11-diene. Metabolic Engineering 2009, 11:13-19.

29. Salis HM, Mirsky EA, Voigt CA: Automated design of synthetic ribosome binding sites to control protein expression. Nature Biotechnology 2009, 27:946-950.

30. Christiansen ME, Znosko BM: Thermodynamic characterization of the complete set of sequence symmetric tandem mismatches in RNA and an improved model for predicting the free energy contribution of sequence asymmetric tandem mismatches. Biochemistry 2008, 47:4329-4336.

31. Na D, Lee D: RBSDesigner: software for designing synthetic ribosome binding sites that yields a desired level of protein expression. Bioinformatics 2010, 26:2633-2634.

32. Nallamsetty S, Waugh DS: Solubility-enhancing proteins MBP and NusA play a passive role in the folding of their fusion partners. Protein Expression and Purification 2006, 45:175-182.

33. Rabhi-Essafi I, Sadok A, Khalaf N, Fathallah DM: A strategy for high-level expression of soluble and functional human interferon alpha as a GST-fusion protein in E.coli. Protein Engineering Design \& Selection 2007, 20:201-209. 
34. Reiling KK, Yoshikuni Y, Martin VJJ, Newman J, Bohlmann J, Keasling JD: Mono and diterpene production in Escherichia coli. Biotechnology and Bioengineering 2004, 87:200-212.

35. Ignea C, Pontini M, Maffei ME, Makris AM, Kampranis SC: Engineering Monoterpene Production in Yeast Using a Synthetic Dominant Negative Geranyl Diphosphate Synthase. Acs Synthetic Biology 2014, 3:298-306.

36. Zhao J, Bao X, Li C, Shen Y, Hou J: Improving monoterpene geraniol production through geranyl diphosphate synthesis regulation in Saccharomyces cerevisiae. Applied Microbiology and Biotechnology 2016, 100:4561-4571.

37. Tashiro M, Kiyota H, Kawai-Noma S, Saito K, Ikeuchi M, lijima Y, Umeno D: Bacterial Production of Pinene by a Laboratory-Evolved Pinene-Synthase. Acs Synthetic Biology 2016, 5:1011-1020.

38. Li J, Perdue E, Pavlostathis S, Araujo RJEi: Physicochemical properties of selected monoterpenes. Environment International 1998, 24:353-358.

\section{Figures}

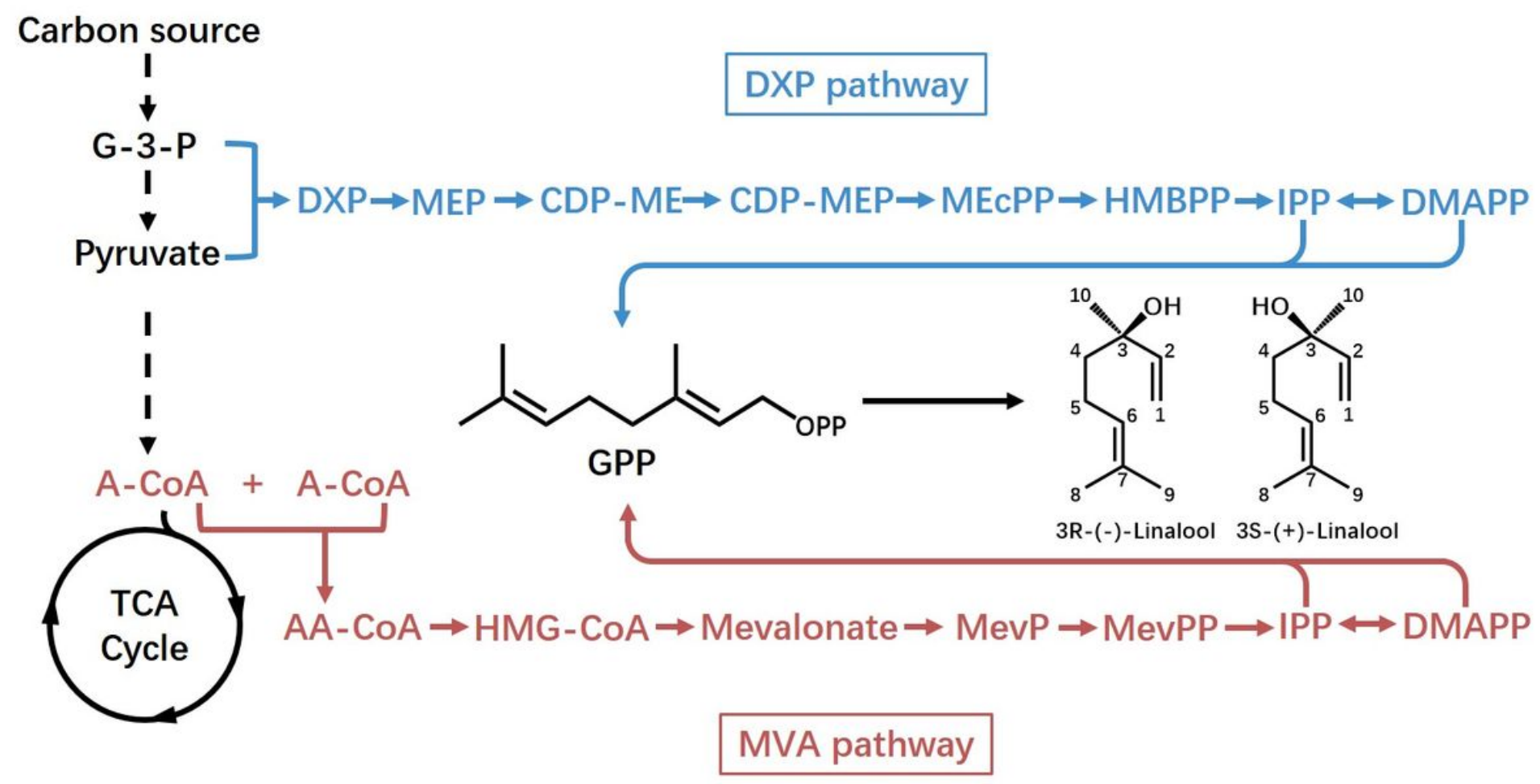

Figure 1

Biosynthesis of linalool via the DXP and MVA pathways. Blue line: endogenous DXP pathway of E. coli. Heterologous MVA pathway indicated by red line. G-3-P: glyceraldehyde 3-phosphate; DXP: 1-deoxy-Dxylulose 5-phosphate; MEP: 2-C-methylerythritol 4-phosphate; CDP-ME: 4-diphosphocytidyl-2-Cmethylerythritol; CDP-MEP: 4-diphosphocytidyl-2-C-methyl-D-erythritol 2-phosphate; MEcPP: 2-C-methyl-Derythritol 2,4-cyclodiphosphate; HMBPP: (E)-4-hydroxy-3-methyl-but-2-enyl-diphosphate; A-CoA: acetylCoA; AA-CoA: acetoacetyl-CoA; HMG-CoA: 3-hydroxy-3-methylglutaryl-CoA; Mev-P, mevalonate-5- 
phosphate; Mev-PP, mevalonate-5-diphosphate; MVA, mevalonate; IPP, isopentenyl pyrophosphate; DMAPP: dimethylallyl diphosphate; GPP: geranyl diphosphate.

a

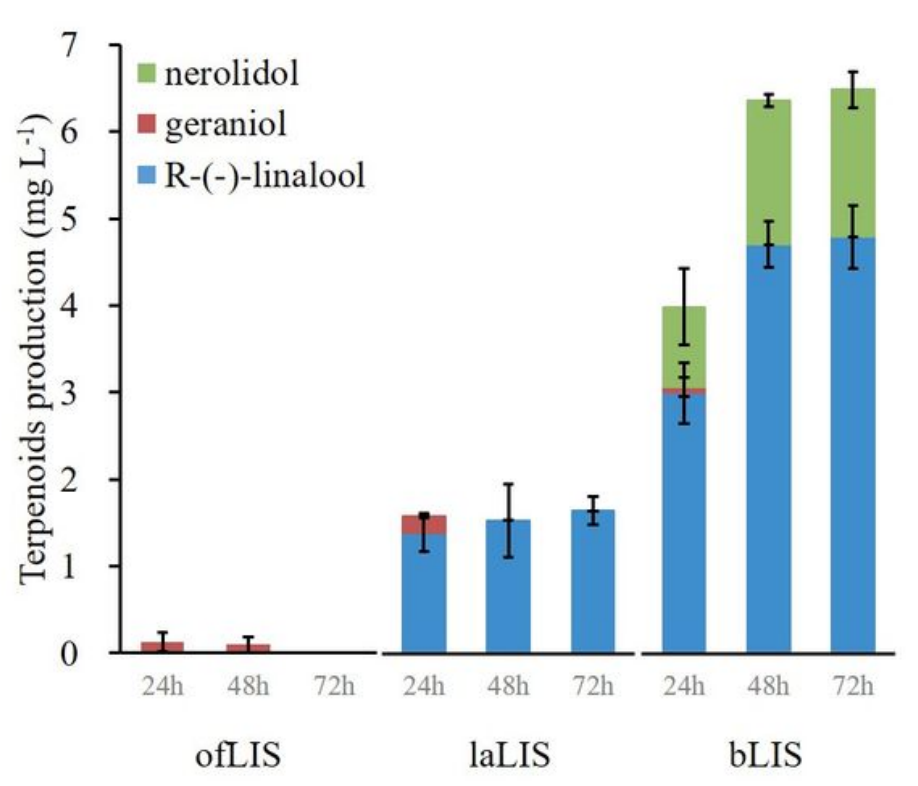

b

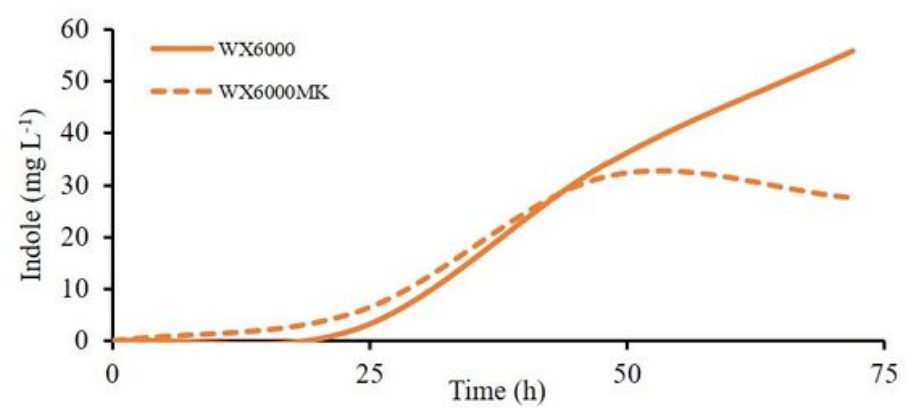

$\mathrm{C}$

R-(-)-linalool production ( $\mathrm{mg} \mathrm{L}^{-1}$ )

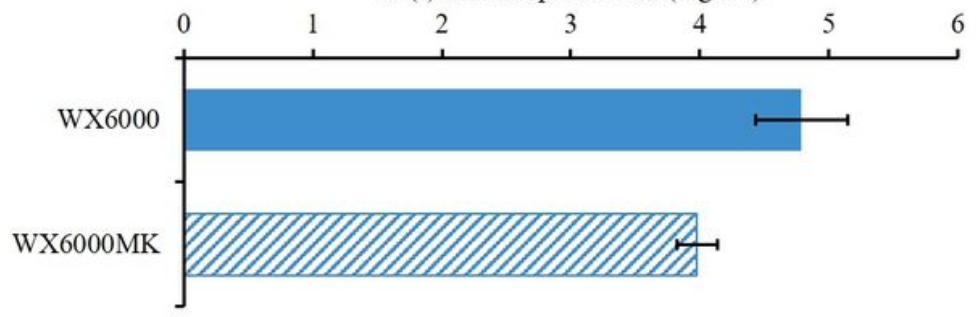

Figure 2

Comparison of recombinant E. coli metabolites. (a) Terpenoids produced by three different LISs at $24 \mathrm{~h}$, $48 \mathrm{~h}$, and $72 \mathrm{~h}$. (b) Comparison of indole titers in the presence and absence of additional exogenous MK. (c) Effect of exogenous MK on R-(-)-linalool production. 
a

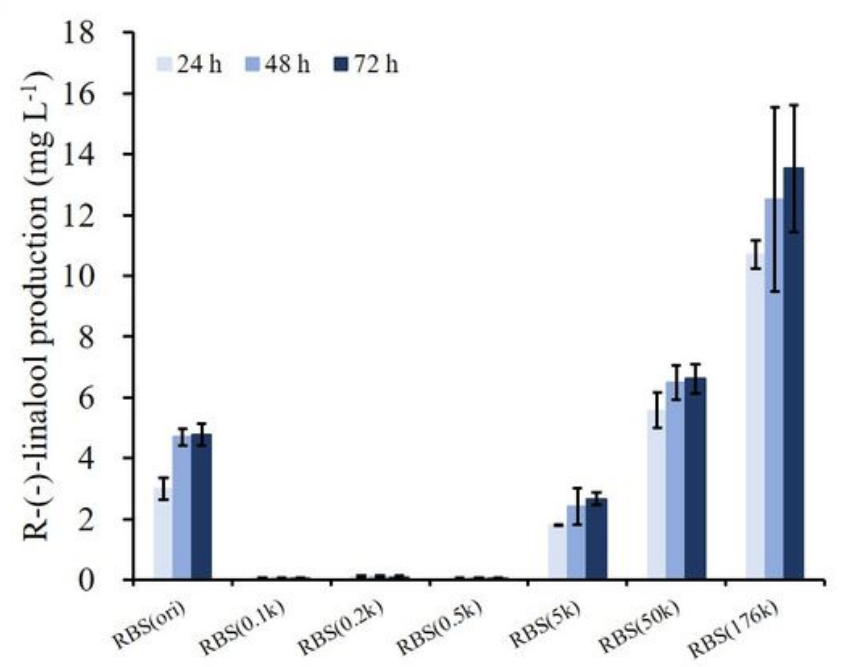

c

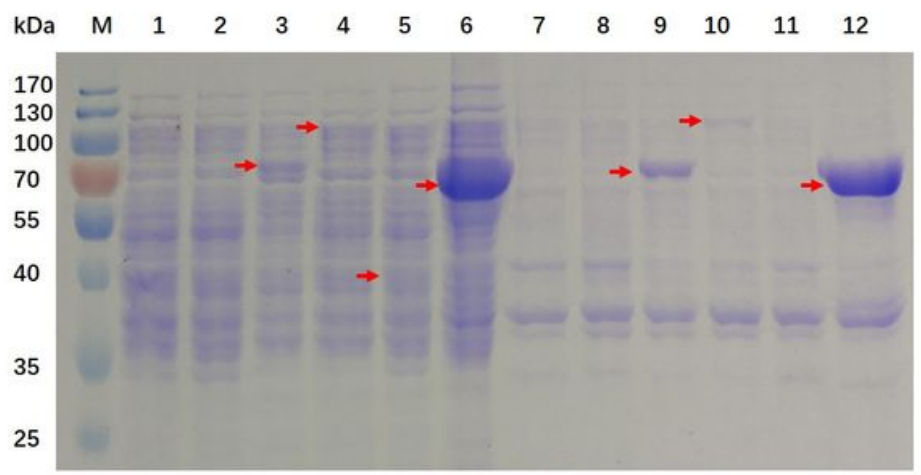

$\mathrm{b}$

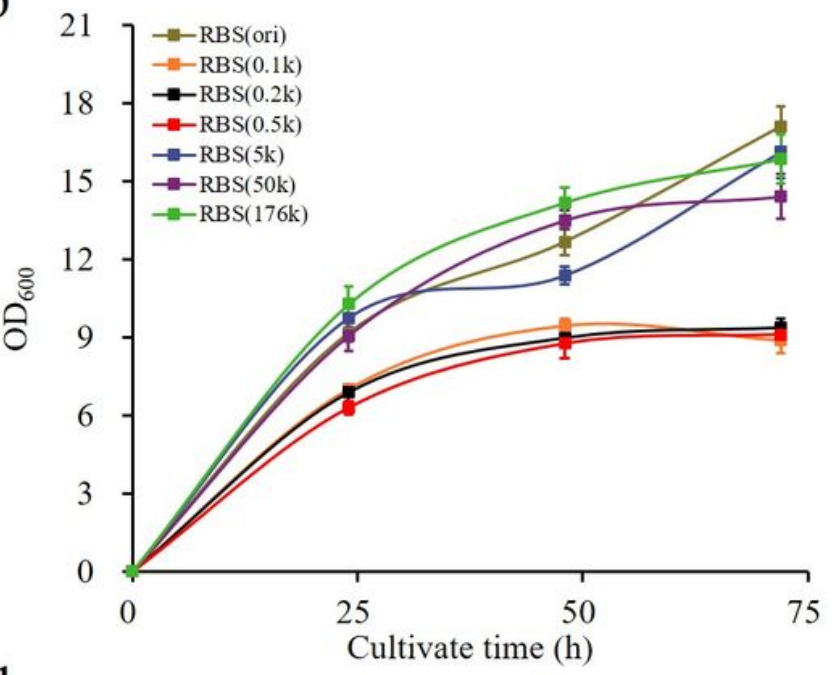

d

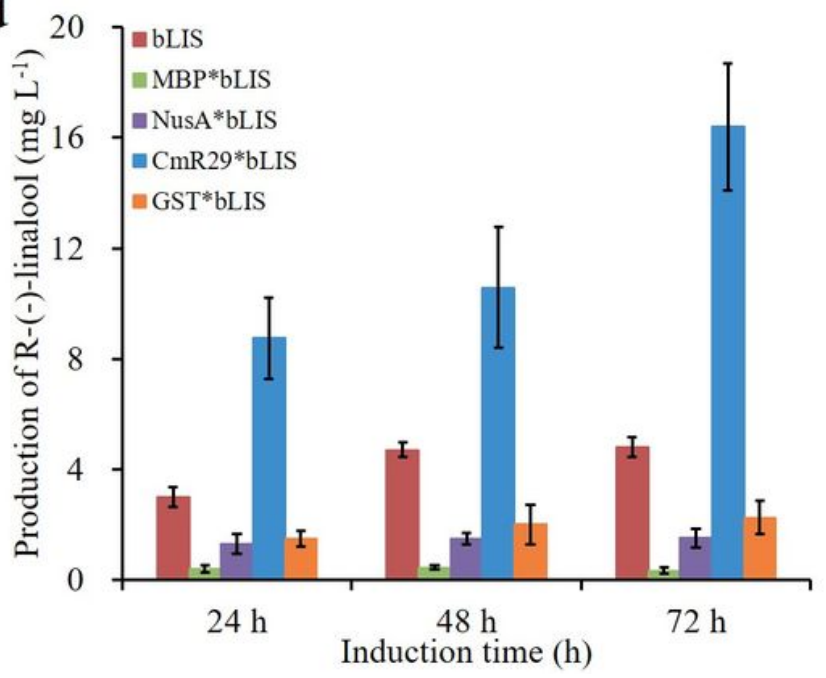

Figure 3

Improvement of R-(-)-linalool production by bLIS expression level modulation. (a) R-(-)-linalool production at $24 \mathrm{~h}, 48 \mathrm{~h}$, and $72 \mathrm{~h}$ by bLIS with various TIR strengths. (b) OD600 for recombinant strains containing different bLIS TIR strengths. (c) SDS-PAGE of wild type and fused bLIS. Red arrows indicate corresponding fused protein. Lane M: molecular weight standard; lane 1: supernatant from control cells; lanes 2-6: supernatants from cells transformed with pETDuet-bLIS, pETDuet-MBP*bLIS, pETDuetNusA*bLIS, pETDuet-CmR29*bLIS, and pETDuet-GST*bLIS, respectively; lane 7: precipitates from control cells; lanes 8-12: precipitates from cells transformed with pETDuet-bLIS, pETDuet-MBP*bLIS, pETDuetNusA*bLIS, pETDuet-CmR29*bLIS, and pETDuet-GST*bLIS, respectively. Gel was stained for total protein with Coomassie Brilliant Blue. (d) R-(-)-linalool production at 24 h, 48 h, and 72 h by bLIS without or with various fusion tags. 
a

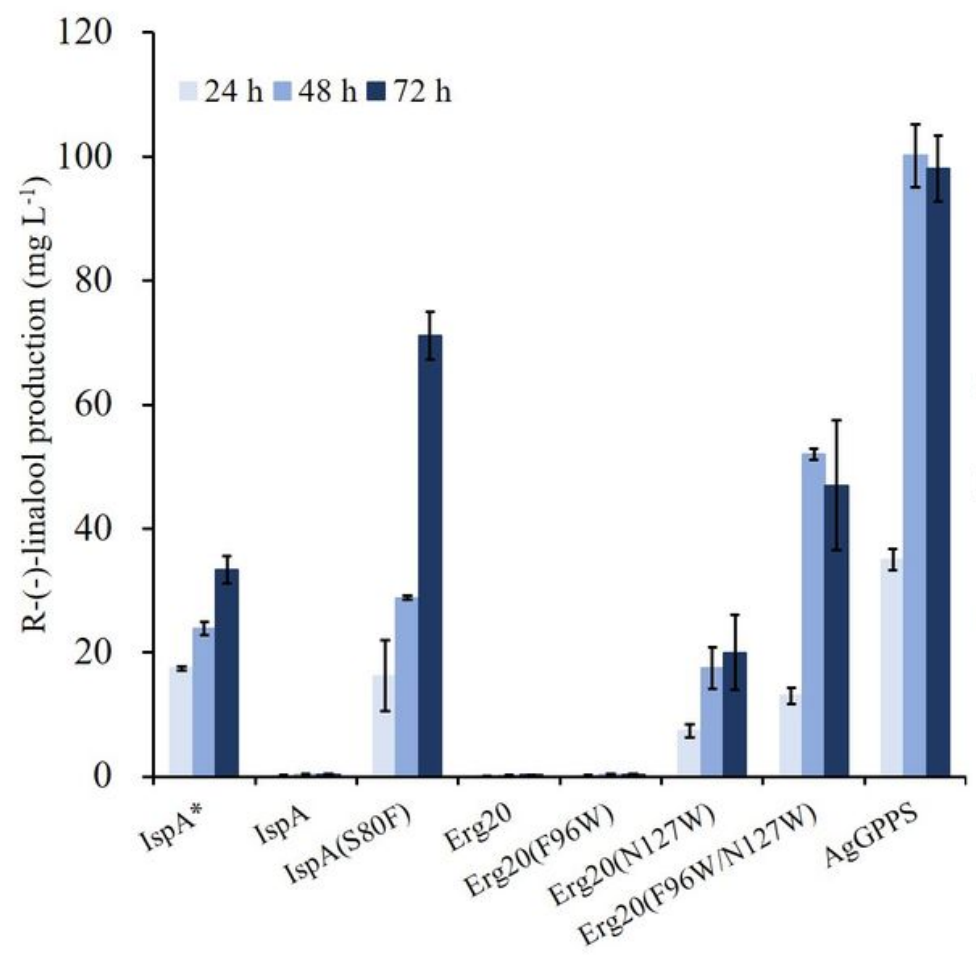

b

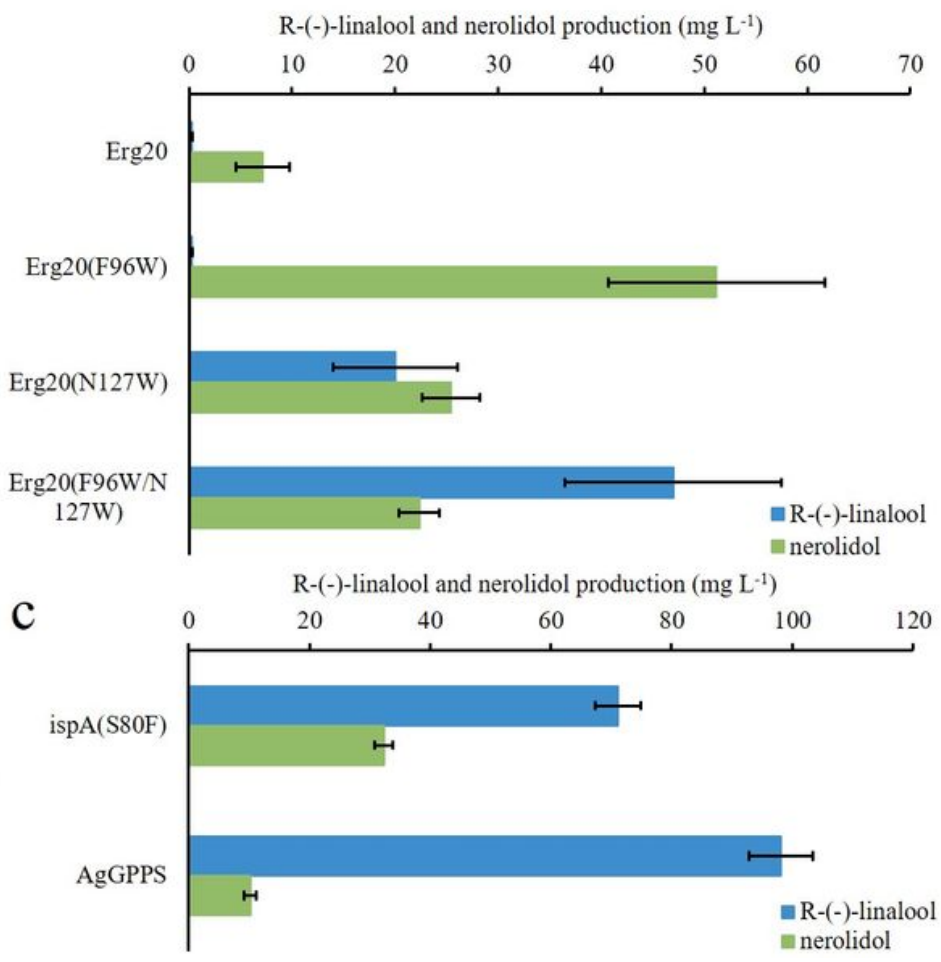

\section{Figure 4}

Effects of various GPPSs on R-(-)-linalool production. Exogenous IspA, IspA (S80F), Erg20, Erg20 (F96W), Erg20 (N127W), Erg20 (F96W/N127W), and AgGPPS were transformed into E. coli. The recombinant strain with its original IspA* served as a control. (a) Effects of different GPPSs on R-(-)-linalool production at $24 \mathrm{~h}, 48 \mathrm{~h}$, and $72 \mathrm{~h}$. (b) Effects of Erg20 and its various mutants on R-(-)-linalool and nerolidol production. (c) Effects of IspA (S80F) and AgGPPS on R-(-)-linalool and nerolidol production. 
a

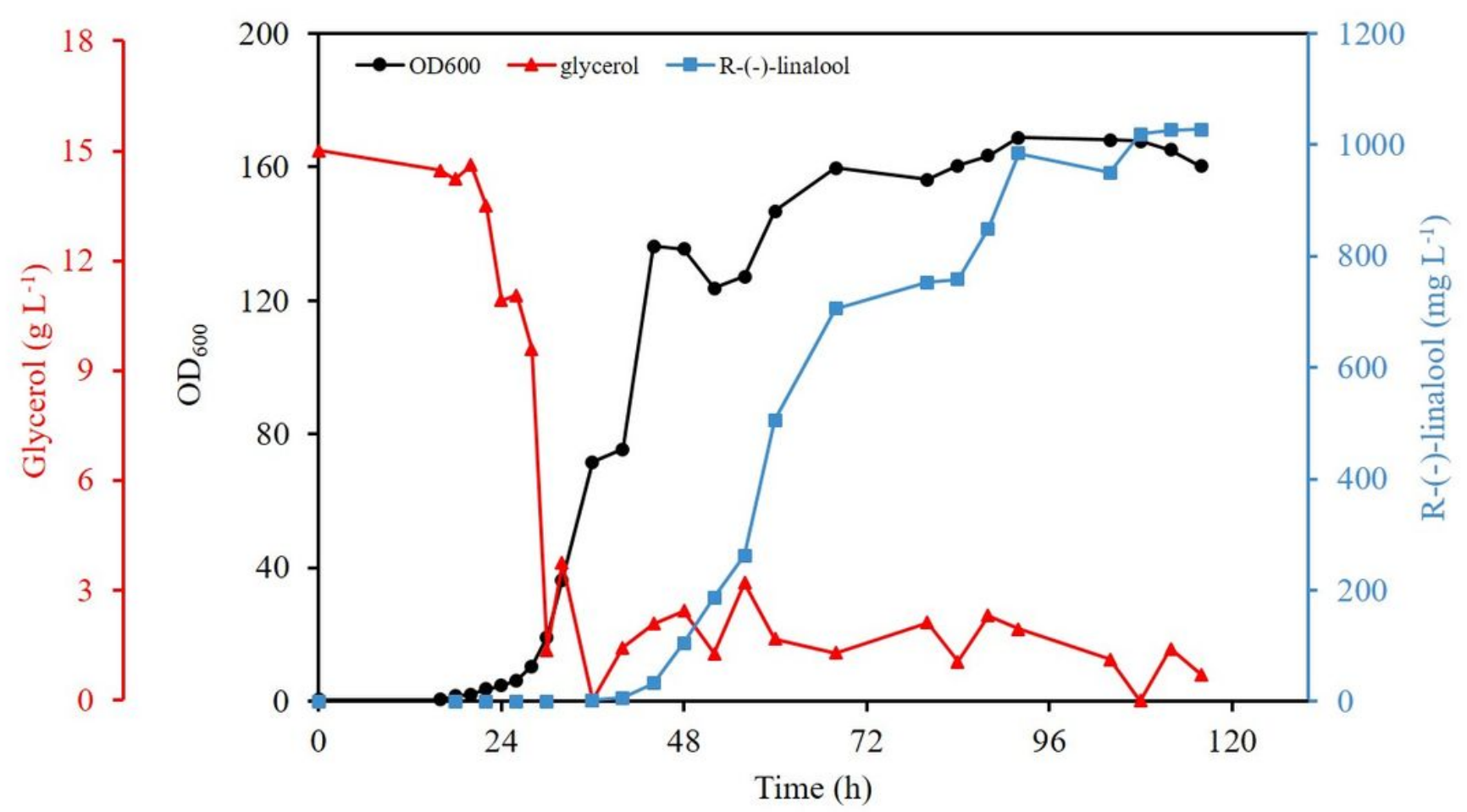

$\mathrm{b}$

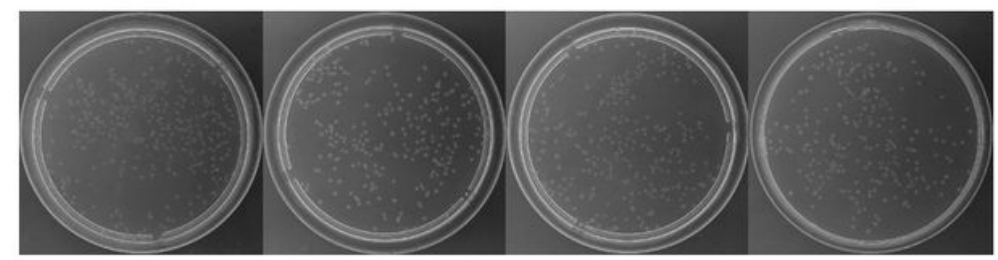

$0 \mathrm{~g} \mathrm{~L}^{-1}$

$1.0 \mathrm{~g} \mathrm{~L}^{-1}$

$1.1 \mathrm{~g} \mathrm{~L}^{-1}$

$1.2 \mathrm{~g} \mathrm{~L}^{-1}$

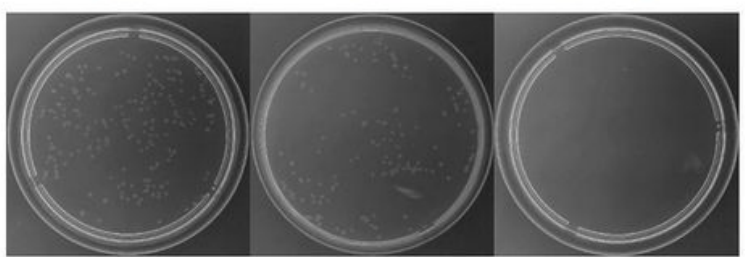
$1.3 \mathrm{~g} \mathrm{~L}^{-1}$
$1.5 \mathrm{~g} \mathrm{~L}^{-1}$

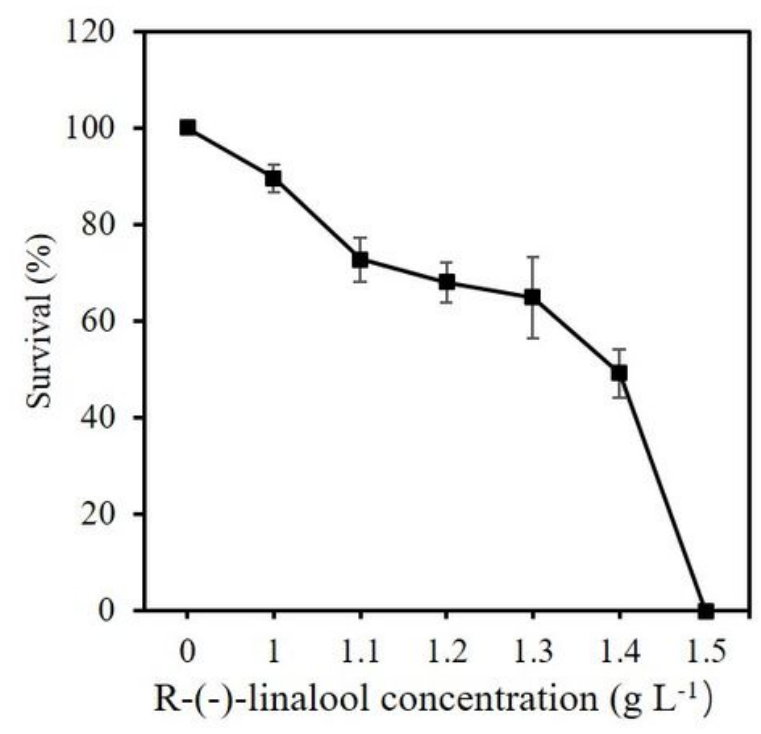

Figure 5

Fed-batch cultivation of engineered E. coli WX6637 in a 1.3-L bioreactor and inhibitory effects of R-(-)linalool. (a) Dark, red, and blue lines represent OD600, glycerol concentration, and R-(-)-linalool production, respectively. (b) Colonies on plates containing various $\mathrm{R}-(-)$-linalool concentrations. (c) E. coli viability in different R-(-)-linalool concentrations. 


\section{R-(-)-linalool increase}

WX4000 (pETDuet-laLIS)

$1.7 \mathrm{mg} \mathrm{L}^{-1} \mathrm{R}-(-)$-linalool

LIS screening

WX6000 (pETDuet-bLIS)

$4.8 \mathrm{mg} \mathrm{L}^{-1} \mathrm{R}$-(-)-linalool
1.0

2.9

Fusion protein overexpression

8.2/9.9

20.4

WX6630 (pETDuet-Cm29*bLIS(max))

$33.4 \mathrm{mg} \mathrm{L}^{-1} \mathrm{R}-(-)-$ linalool

GPPS mutation and screening

\section{WX6637 (pETDuet-Cm29*bLIS(max)-AgGPPS)}

$100.1 \mathrm{mg} \mathrm{L}^{-1} \mathrm{R}-(-)$-linalool

60.7

Fed-batch fermentation

WX6637 (pETDuet-Cm29*bLIS(max)-AgGPPS)

$1027.3 \mathrm{mg} \mathrm{L}^{-1} \mathrm{R}$-(-)-linalool

622.6

\section{Figure 6}

A systematic approach for metabolic engineering of E. coli to produce the acyclic monoterpene R-(-)linalool.

\section{Supplementary Files}

This is a list of supplementary files associated with this preprint. Click to download.

- Additionalfile1.docx 